\title{
GENERALIZED PREINVEX FUNCTIONS AND THEIR PROPERTIES
}

\author{
MUHAMMAD ASLAM NOOR AND KHALIDA INAYAT NOOR
}

Received 9 January 2005; Revised 9 August 2005; Accepted 2 September 2005

We introduce some new classes of preinvex and invex functions, which are called $\varphi$ preinvex and $\varphi$-invex functions. We study some properties of these classes of $\varphi$-preinvex ( $\varphi$-invex) functions. In particular, we establish the equivalence among the $\varphi$-preinvex functions, $\varphi$-invex functions, and $\varphi \eta$-monotonicity of their differential under some suitable conditions.

Copyright (c) 2006 M. A. Noor and K. I. Noor. This is an open access article distributed under the Creative Commons Attribution License, which permits unrestricted use, distribution, and reproduction in any medium, provided the original work is properly cited.

\section{Introduction}

In recent years, several extensions and generalizations have been considered for classical convexity. A significant generalization of convex functions is that of invex functions introduced by Hanson [1]. Hanson's initial result inspired a great deal of subsequent work which has greatly expanded the role and applications of invexity in nonlinear optimization and other branches of pure and applied sciences. Weir and Mond [8] and Noor [3, 4] have studied the basic properties of the preinvex functions and their role in optimization and mathematical programming problems. It is well known that the preinvex functions and invex sets may not be convex functions and convex sets. Equally important is another generalization of the convex function which is called $\varphi$-convex function introduced and studied by Noor [5]. In particular, these generalizations of the convex functions are quite different and do not contain each other. In this paper, we introduce and considered another class of nonconvex functions, which include these generalizations as special cases. This class of nonconvex functions is called the $\varphi$-preinvex and $\varphi$-invex functions. Several new concepts of $\varphi \eta$-monotonicity are introduced. We establish the relationship between these classes and derive some new results. As special cases, one can obtain some new and correct versions of known results. Results obtained in this paper present a refinement and improvement of previously known results. 
2 Generalized preinvex functions and their properties

\section{Preliminaries}

Let $K$ be a nonempty closed set in a real Hilbert space $H$. We denote by $\langle\cdot, \cdot\rangle$ and $\|\cdot\|$ the inner product and norm, respectively. Let $F: K \rightarrow H$ and $\eta(\cdot, \cdot): K \times K \rightarrow R$ be continuous functions. Let $\varphi: K \rightarrow R$ be a continuous function.

Definition 2.1. Let $u \in K$. Then the set $K$ is said to be $\varphi$-invex at $u$ with respect to $\eta(\cdot, \cdot)$ and $\varphi(\cdot)$, if

$$
u+t e^{i \varphi} \alpha(v, u) \eta(v, u) \in K, \quad \forall u, v \in K, t \in[0,1]
$$

$K$ is said to be a $\varphi$-invex set with respect to $\eta$ and $\varphi$, if $K$ is $\varphi$-invex at each $u \in K$. The $\varphi$-invex set $K$ is also called $\varphi \eta$-connected set. Note that the convex set with $\varphi=0$ and $\eta(v, u)=v-u$ is a $\varphi$-invex set, but the converse is not true. For example, the set $K=$ $R-(-1 / 2,1 / 2)$ is a $\varphi$-invex set with respect to $\eta$ and $\varphi=0$, where

$$
\eta(v, u)= \begin{cases}v-u, & \text { for } v>0, u>0 \text { or } v<0, u<0 \\ u-v, & \text { for } v<0, u>0 \text { or } v<0, u<0\end{cases}
$$

It is clear that $K$ is not a convex set.

Remark 2.2. (i) If $\varphi=0$, then the set $K$ is called the invex ( $\eta$-connected) set, see $[2,3,8-$ 11].

(ii) If $\eta(v, u)=v-u$, then the set $K$ is called the $\varphi$-convex set, see Noor [5].

(iii) If $\varphi=0$ and $\eta(v, u)=v-u$, then the set $K$ is called the convex set.

From now onward $K$ is a nonempty closed $\varphi$-invex set in $H$ with respect to $\varphi$ and $\eta(\cdot, \cdot)$, unless otherwise specified.

Definition 2.3. The function $F$ on the $\varphi$-invex set $K$ is said to be $\varphi$-preinvex with respect to $\eta$ and $\varphi$, if

$$
F\left(u+t e^{i \varphi} \eta(v, u)\right) \leq(1-t) F(u)+t F(v), \quad \forall u, v \in K, t \in[0,1] .
$$

The function $F$ is said to be $\varphi$-preconcave if and only if $-F$ is $\varphi$-preinvex. Note that every convex function is a $\varphi$-preinvex function, but the converse is not true. For example, the function $F(u)=-|u|$ is not a convex function, but it is a $\varphi$-preinvex function with respect to $\eta$ and $\varphi=0$, where

$$
\eta(v, u)= \begin{cases}v-u & \text { if } v \leq 0, u \leq 0, v \geq 0, u \geq 0 \\ u-v & \text { otherwise. }\end{cases}
$$

Definition 2.4. The function $F$ on the $\varphi$-invex set $K$ is called quasi- $\varphi$-preinvex with respect to $\varphi$ and $\eta$, if

$$
F\left(u+t e^{i \varphi} \eta(v, u)\right) \leq \max \{F(u), F(v)\}, \quad \forall u, v \in K, t \in[0,1] .
$$


Definition 2.5. The function $F$ on the $\varphi$-invex set $K$ is said to be logarithmic $\varphi$-preinvex with respect to $\varphi$ and $\eta$, if

$$
F\left(u+t e^{i \varphi} \eta(v, u)\right) \leq(F(u))^{1-t}(F(v))^{t}, \quad u, v \in K, t \in[0,1],
$$

where $F(\cdot)>0$.

From the above definitions,

$$
\begin{aligned}
F\left(u+t e^{i \varphi} \eta(v, u)\right) & \leq(F(u))^{1-t}(F(v))^{t} \\
& \leq(1-t) F(u)+t F(v) \\
& \leq \max \{F(u), F(v)\} \\
& <\max \{F(u), F(v)\} .
\end{aligned}
$$

For $t=1$, Definitions 2.3 and 2.5 reduce to the following condition.

Condition 2.6.

$$
F\left(u+e^{i \varphi} \eta(v, u)\right) \leq F(v), \quad \forall u, v \in K
$$

which plays an important part in studying the properties of the $\varphi$-preinvex $(\varphi$-invex) functions.

For $\varphi=0$, Condition 2.6 reduces to the following for preinvex functions.

Condition 2.7.

$$
F(u+\eta(v, u)) \leq F(v), \quad \forall u, v \in K
$$

For the applications of Condition 2.7, see [4, 8-11].

Definition 2.8. The function $F$ on the $\varphi$-invex set $K$ is said to be pseudo- $\varphi$-preinvex with respect to $\varphi$ and $\eta$, if there exists a strictly positive function $b(\cdot, \cdot)$ such that

$$
F(v) \leq F(u) \Longrightarrow F\left(u+t e^{i \varphi} \eta(v, u)\right) \leq F(u)+t(t-1) b(u, v), \quad u, v \in K, t \in[0,1] .
$$

Lemma 2.9. If the function $F$ is $\varphi$-preinvex function with respect to $\varphi$ and $\eta$, then $F$ is pseudo- $\varphi$-preinvex function with respect to $\varphi$ and $\eta$.

Proof. Without loss of generality, we assume that $F(v)<F(u)$, for all $u, v \in K$. For every $t \in[0,1]$, we have

$$
\begin{aligned}
F\left(u+t e^{i \varphi} \eta(v, u)\right) & \leq(1-t) F(u)+t F(v) \\
& <F(u)+t(t-1)\{F(u)-F(v)\} \\
& =F(u)+t(t-1) b(v, u),
\end{aligned}
$$

where $b(v, u)=F(v)-F(u)>0$.

Thus, it follows that the function $F$ is pseudo- $\varphi$-preinvex function with respect to $\varphi$ and $\eta$, the required result. 
4 Generalized preinvex functions and their properties

Lemma 2.10. Let $F$ be a $\varphi$-preinvex function. Then any local minimum of $F$ on $K$ is a global minimum.

Proof. Let the $\varphi$-preinvex function $F$ have a local minimum at $u \in K$. Assume the contrary, that is, $F(v)<F(u)$ for some $v \in K$. Since $F$ is a $\varphi$-preinvex function, so

$$
F\left(u+t e^{i \varphi} \eta(v, u)\right) \leq F(u)+t(F(v)-F(u)),
$$

which implies that

$$
F\left(u+t e^{i \varphi} \eta(v, u)\right)-F(u)<0,
$$

for arbitrary small $t>0$, contradicting the local minimum.

Essentially using the technique and ideas of the classical convexity, one can easily prove the following results.

Theorem 2.11. If $F$ is a $\varphi$-preinvex function on $K$, then the level set $L_{\alpha}=\{u \in K: F(u) \leq$ $\alpha, \alpha \in R\}$ is a $\varphi$-invex set with respect to $\varphi$ and $\eta$.

Theorem 2.12. The function $F$ is a $\varphi$-preinvex function if and only if epi $(F)=\{(u, \alpha): u \in$ $K, \alpha \in R, F(u) \leq \alpha$ \} is a $\varphi$-invex set with respect to $\varphi$ and $\eta$.

Theorem 2.13. The function $F$ is a quasi- $\varphi$-convex function if and only if the level set $L_{\alpha}=\{u \in K, \alpha \in R: f(u) \leq \alpha\}$ is a $\varphi$-invex set with respect to $\varphi$ and $\eta$.

Theorem 2.14. Let $F$ be a $\varphi$-preinvex function with respect to $\varphi$ and $\eta$. If $\phi: L \rightarrow R$ is a nondecreasing function, then $\phi \circ F$ is a $\varphi$-preinvex function with respect to the function $\varphi$ and $\eta$.

Proof. Since $F$ is a $\varphi$-preinvex function and $\phi$ is decreasing, we have that for all $u, v \in K$ and $t \in[0,1]$,

$$
\begin{aligned}
\phi \circ F\left(u+t e^{i \varphi} \eta(v, u)\right) & \leq \phi\left[F\left(u+t e^{i \varphi} \eta(v, u)\right)\right] \\
& \leq \phi[(1-t)(F u)+t F(v)] \\
& \leq(1-t) \phi \circ F(u)+\phi \circ F(v),
\end{aligned}
$$

from which it follows that $\phi \circ F$ is a $\varphi$-convex function with respect to $\varphi$ and $\eta$.

Definition 2.15. The differentiable function $F$ on the $\varphi$-invex set $K$ is said to be a $\varphi$-invex function with respect to $\varphi$ and $\eta(\cdot, \cdot)$, if

$$
F(v)-F(u) \geq\left\langle F_{\varphi}^{\prime}(u), \eta(v, u)\right\rangle, \quad \forall u, v \in K,
$$

where $F_{\varphi}^{\prime}(u)$ is the differential of $F$ at $u$ in the direction of $v-u \in K$. Note that for $\varphi=$ 0 , we obtain the original definition of invexity which is due to Hanson [1]. It is well known that the concepts of preinvex and invex functions play a significant role in the mathematical programming and optimization theory, see $[3,6,8-11]$ and the references therein. 
Definition 2.16. An operator $T: K \rightarrow H$ is said to be

(i) strongly $\eta$-monotone if and only if there exists a constant $\alpha>0$ such that

$$
\langle T u, \eta(v, u)\rangle+\langle T v, \eta(u, v)\rangle \leq-\alpha\left\{\|\eta(v, u)\|^{2}+\|\eta(u, v)\|^{2}\right\}, \quad \forall u, v \in K
$$

(ii) $\eta$-monotone if and only if

$$
\langle T u, \eta(v, u)\rangle+\langle T v, \eta(u, v)\rangle \leq 0, \quad \forall u, v \in K
$$

(iii) strongly $\eta$-pseudomonotone, if and only if, there exists a constant $v>0$ such that

$$
\langle T u, \eta(v, u)\rangle+v\|\eta(v, u)\|^{2} \geq 0 \Longrightarrow-\langle T v, \eta(u, v)\rangle \geq 0, \quad \forall u, v \in K
$$

(iv) strictly $\eta$-monotone if and only if

$$
\langle T u, \eta(v, u)\rangle+\langle T v, \eta(u, v)\rangle<0, \quad \forall u, v \in K
$$

(v) $\eta$-pseudomonotone if and only if

$$
\langle T u, \eta(v, u)\rangle \geq 0 \Longrightarrow\langle T v, \eta(u, v)\rangle \leq 0, \quad \forall u, v \in K
$$

(vi) quasi- $\eta$-monotone if and only if

$$
\langle T u, \eta(v, u)\rangle>0 \Longrightarrow\langle T v, \eta(u, v)\rangle \leq 0, \quad \forall u, v \in K
$$

(vii) strictly $\eta$-pseudomonotone if and only if

$$
\langle T u, \eta(v, u)\rangle \geq 0 \Longrightarrow\langle T v, \eta(u, v)\rangle<0, \quad \forall u, v \in K .
$$

Note for $\varphi=0$, for all $u, v \in K$, the $\varphi$-invex set $K$ becomes an invex set. In this case, Definition 2.16 is exactly the same as in $[6,7,10,11]$. In addition, if $\varphi=0$ and $\eta(v, u)=$ $v-u$, then the $\varphi$-invex set $K$ is the convex set $K$, and consequently Definition 2.16 reduces to the one in [7] for the convex set $K$. This clearly shows that Definition 2.16 is more general than the ones in $[6,7,10,11]$ and includes them as special cases.

Definition 2.17. A differentiable function $F$ on a $\varphi$-invex set $K$ is said to be strongly pseudo- $\varphi \eta$-invex function if and only if there exists a constant $\mu>0$ such that

$$
\left\langle F_{\varphi}^{\prime}(u), \eta(v, u)\right\rangle+\mu\|\eta(u, v)\|^{2} \geq 0 \Longrightarrow F(v)-F(u) \geq 0, \quad \forall u, v \in K .
$$

Definition 2.18. A differentiable function $F$ on the $K$ is said to be strongly quasi- $\varphi$-invex if there exists a constant $\mu>0$ such that

$$
F(v) \leq F(u) \Longrightarrow\left\langle F_{\varphi}^{\prime}(u), \eta(v, u)\right\rangle+\mu\|\eta(v, u)\|^{2} \leq 0, \quad \forall u, v \in K .
$$

Definition 2.19. The function $F$ on the set $K$ is said to be pseudo- $\alpha$-invex if

$$
\left\langle F_{\varphi}^{\prime}(u), \eta(v, u)\right\rangle \geq 0 \Longrightarrow F(v) \geq F(u), \quad \forall u, v \in K .
$$


6 Generalized preinvex functions and their properties

Definition 2.20. A differentiable function $F$ on the set $K$ is said to be quasi- $\varphi$-invex if such that

$$
F(v) \leq F(u) \Longrightarrow\left\langle F_{\varphi}^{\prime}(u), \eta(v, u)\right\rangle \leq 0, \quad \forall u, v \in K
$$

Note that if $\varphi=0$, then the $\varphi$-invex set $K$ is exactly the invex set $K$, and consequently Definitions 2.17-2.19 are exactly the same as in $[6,11]$. In particular, if $\varphi=0$ and $\eta(v, u)=$ $-\eta(v, u)$, for all $u, v \in K$, that is, the function $\eta(\cdot, \cdot)$ is skew-symmetric, then Definitions $2.16-2.19$ reduce to the ones in $[6,11]$. This shows that the concepts introduced in this paper represent an improvement of the previously known ones. All the concepts defined above play an important and fundamental part in the mathematical programming and optimization problems.

We also need the following assumption regarding the function $\eta(\cdot, \cdot)$, and $\varphi$.

Condition 2.21. Let $\eta(\cdot, \cdot): K \times K \rightarrow H$ and let $\varphi$ satisfy the assumptions

$$
\begin{gathered}
\eta\left(u, u+t e^{i \varphi} \eta(v, u)\right)=-t \eta(v, u), \\
\eta\left(v, u+t e^{i \varphi} \eta(v, u)\right)=(1-t) \eta(v, u), \quad \forall u, v \in K, t \in[0,1] .
\end{gathered}
$$

Clearly for $t=0$, we have $\eta(u, v)=0$ if and only if $u=v$, for all $u, v \in K$. One can easily show $[9,11]$ that $\eta\left(u+t e^{i \varphi} \eta(v, u), u\right)=t \eta(v, u)$, for all $u, v \in K$.

Note that for $\varphi=0$, Condition 2.21 collapses to the following condition, which is due to Mohan and Neogy [2].

Condition 2.22. Let $\eta(\cdot, \cdot): K \times K \rightarrow H$ satisfy the assumptions

$$
\begin{gathered}
\eta\left(u, u+t e^{i \varphi} \eta(v, u)\right)=-t \eta(v, u), \\
\eta\left(v, u+t e^{i \varphi} \eta(v, u)\right)=(1-t) \eta(v, u), \quad \forall u, v \in K, t \in[0,1] .
\end{gathered}
$$

For the applications of Condition 2.22, see $[2,4,9,11]$.

\section{Main results}

In this section, we study some basic properties of $\varphi$-preinvex functions on the $\varphi$-invex set $K$.

Theorem 3.1. Let $F$ be a differentiable function on the $\varphi$-invex set $K$ and let Condition 2.21 hold. Then the function $F$ is a $\varphi$-preinvex function if and only if $F$ is a $\varphi$-invex function.

Proof. Let $F$ be a $\varphi$-preinvex function on the set $K$. Then, for all $u, v \in K, t \in[0,1]$, $u+t e^{i \varphi} \eta(v, u) \in K$ and

$$
F\left(u+t e^{i \varphi} \eta(v, u)\right) \leq(1-t) F(u)+t F(v), \quad \forall u, v \in K,
$$

which can be written as

$$
F(v)-F(u) \geq \frac{F\left(u+t e^{i \varphi} \eta(v, u)\right)-F(u)}{t} .
$$


Letting $t \rightarrow 0$ in the above inequality, we have

$$
F(v)-F(u) \geq\left\langle F_{\varphi}^{\prime}(u), \eta(v, u)\right\rangle,
$$

which implies that $F$ is a $\varphi$-invex function.

Conversely, let $F$ be a $\varphi$-invex function on the $\varphi$-invex function $K$. Then for all $u, v \in K$, $t \in[0,1], v_{t}=u+t e^{i \varphi} \eta(v, u) \in K$, and using Condition 2.21, we have

$$
\begin{aligned}
F(v) & -F\left(u+t e^{i \varphi} \eta(v, u)\right) \\
& \geq\left\langle F_{\varphi}^{\prime}\left(u+t e^{i \varphi} \eta(v, u)\right), \eta\left(v, u+t e^{i \varphi} \eta(v, u)\right)\right\rangle \\
& =(1-t)\left\langle F_{\varphi}^{\prime}(u+t \alpha(v, u) \eta(v, u)), \eta(v, u)\right\rangle .
\end{aligned}
$$

In a similar way, we have

$$
\begin{aligned}
F(u) & -F\left(u+t e^{i \varphi} \eta(v, u)\right) \\
& \geq\left\langle F_{\varphi}^{\prime}\left(u+t e^{i \varphi} \eta(v, u)\right), \eta\left(u, u+t e^{i \varphi} \eta(v, u)\right)\right\rangle \\
& =-t\left\langle F_{\varphi}^{\prime}\left(u+t e^{i \varphi} \eta(v, u)\right), \eta(v, u)\right\rangle .
\end{aligned}
$$

Multiplying (3.4) by $t$ and (3.5) by $(1-t)$, and adding the resultant, we have

$$
F\left(u+t e^{i \varphi} \eta(v, u)\right) \leq(1-t) F(u)+t F(v),
$$

showing that $F$ is a $\varphi$-preinvex function.

If $\varphi=0$, then Theorem 3.1 reduces to the following result of Mohan and Neogy [2] for the preinvex and invex functions on the invex set.

Theorem 3.2. Let $F$ be a differentiable function on the invex set $K$ and let Condition 2.22 hold. Then the function $F$ is a preinvex function if and only if $F$ is an invex function.

Theorem 3.3. Let $F$ be a differentiable function on the invex set $K$ and let Condition 2.6 hold. Then its differential $F_{\varphi}^{\prime}(u)$ is $\varphi \eta$-monotone if and only if $F$ is $\varphi$-invex ( $\varphi$-preinvex) function on $K$.

Proof. Let $F$ be a $\varphi$-invex function on $K$. Then

$$
F(v)-F(u) \geq\left\langle F_{\varphi}^{\prime}(u), \eta(v, u)\right\rangle, \quad \forall u, v \in K
$$

Changing the role of $u$ and $v$ in (3.7), we have

$$
F(u)-F(v) \geq\left\langle F_{\varphi}^{\prime}(v), \eta(u, v)\right\rangle, \quad \forall u, v \in K
$$

Adding (3.7) and (3.8), we have

$$
\left\langle F_{\varphi}^{\prime}(u), \eta(v, u)\right\rangle+\left\langle F_{\varphi}^{\prime}(v), \eta(u, v)\right\rangle \leq 0,
$$

which shows that $F_{\varphi}^{\prime}$ is $\varphi \eta$-monotone. 
8 Generalized preinvex functions and their properties

Conversely, let $F_{\varphi}^{\prime}(u)$ be $\varphi \eta$-monotone, that is,

$$
\left\langle F_{\varphi}^{\prime}(v), \eta(u, v)\right\rangle+\left\langle F_{\varphi}^{\prime}(u), \eta(v, u)\right\rangle \leq 0, \quad \forall u, v \in K
$$

which implies that

$$
\left\langle F^{\prime}(v), \eta(u, v)\right\rangle \leq-\left\langle F^{\prime}(u), \eta(v, u)\right\rangle
$$

Since $K$ is a $\varphi$-invex set, for all $u, v \in K, t \in[0,1], v_{t}=u+t e^{i \varphi} \eta(v, u) \in K$. Taking $v \equiv v_{t}$ in (3.11) and using Condition 2.21, we have

$$
-t\left\langle F_{\varphi}^{\prime}\left(u+t e^{i \varphi} \eta(v, u)\right), \eta(v, u)\right\rangle \leq-t\left\langle F_{\varphi}^{\prime}(u), \eta(v, u)\right\rangle,
$$

which implies that

$$
\left\langle F_{\varphi}^{\prime}(u+t \alpha(v, u) \eta(v, u)), \eta(v, u)\right\rangle \geq\left\langle F^{\prime}\left({ }_{\varphi} u\right), \eta(v, u)\right\rangle .
$$

Let

$$
g(t)=F\left(u+t e^{i \varphi} \eta(v, u)\right), \quad \forall u, v \in K, t \in[0,1] .
$$

Then, using (3.13), we have

$$
\begin{aligned}
g^{\prime}(t) & =\left\langle F_{\varphi}^{\prime}\left(u+t e^{i \varphi} \eta(v, u)\right), \eta(v, u)\right\rangle \\
& \geq\left\langle F_{\varphi}^{\prime}(u), \eta(v, u)\right\rangle .
\end{aligned}
$$

Integrating the above relation between 0 and 1 , we have

$$
g(1)-g(0) \geq\left\langle F_{\varphi}^{\prime}(u), \eta(v, u)\right\rangle
$$

that is,

$$
F\left(u+e^{i \varphi} \eta(v, u)\right)-F(u) \geq\left\langle F_{\varphi}^{\prime}(u), \eta(v, u)\right\rangle,
$$

which implies, using Condition 2.6, that

$$
F(v)-F(u) \geq\left\langle F_{\varphi}^{\prime}(u), \eta(v, u)\right\rangle
$$

which shows that the function $F(u)$ is a $\varphi$-invex ( $\alpha$-preinvex) function, the required result.

For $\varphi=0$, the $\alpha$-invex set $K$ becomes the invex set, and consequently we have following result for preinvex and invex functions. 
Theorem 3.4. Let Conditions 2.7 and 2.22 hold and let $K$ be an invex set. Then the differential $F^{\prime}(u)$ of a function $F(u)$ is $\eta$-monotone if and only if $F(u)$ is an invex(preinvex) function on the invex set $K$.

We now give a necessary condition for strongly $\varphi \eta$-pseudoinvex function, which is also a generalization and refinement of a result proved in $[6,11]$.

Theorem 3.5. Let the differential $F_{\varphi}^{\prime}(u)$ of a function $F(u)$ on the $\varphi$-invex set $K$ be strongly $\varphi \eta$-pseudomonotone. If Conditions 2.6 and 2.21 hold, then $F$ is strongly pseudo- $\varphi \eta$-invex function.

Proof. Let $F_{\varphi}^{\prime}(u)$ be strongly $\varphi \eta$-pseudomonotone. Then

$$
\left\langle F_{\varphi}^{\prime}(u), \eta(v, u)\right\rangle+\mu\|\eta(v, u)\|^{2} \geq 0, \quad \forall u, v \in K,
$$

implies that

$$
-\left\langle F_{\varphi}^{\prime}(v), \eta(u, v)\right\rangle \geq 0, \quad \forall u, v \in K
$$

Since $K$ is a $\varphi$-invex set, for all $u, v \in K, t \in[0,1], v_{t}=u+t e^{i \varphi} \eta(v, u) \in K$. Taking $v=v_{t}$ in (3.20) and using Condition 2.21, we have

$$
\left\langle F_{\varphi}^{\prime}\left(u+t e^{i \varphi} \eta(v, u)\right), \eta(v, u)\right\rangle \geq 0, \quad \forall u, v \in K
$$

Let

$$
g(t)=F\left(u+t e^{i \varphi} \eta(v, u)\right), \quad \forall u, v \in K, t \in[0,1] .
$$

Then, using (3.21), we have

$$
g^{\prime}(t)=\left\langle F_{\varphi}^{\prime}\left(u+t e^{i \varphi} \eta(v, u)\right), \eta(v, u)\right\rangle \geq 0 .
$$

Integrating the above relation between 0 and 1 , we have

$$
g(1)-g(0) \geq 0
$$

that is,

$$
F\left(u+e^{i \varphi} \eta(v, u)\right)-F(u) \geq 0,
$$

which implies, using Condition 2.6, that

$$
F(v)-F(u) \geq 0
$$

showing that the function $F(u)$ is strongly pseudo- $\varphi \eta$-invex function. 
10 Generalized preinvex functions and their properties

As special cases of Theorem 3.5, we have the following.

Theorem 3.6. Let the differential $F_{\varphi}^{\prime}(u)$ of a function $F(u)$ on the $\varphi$-invex set $K$ be $\varphi \eta$ pseudomonotone. If Conditions 2.6 and 2.21 hold, then $F$ is pseudo- $\varphi \eta$-invex function.

Theorem 3.7. Let the differential $F_{\varphi}^{\prime}(u)$ of a function $F(u)$ on the invex set $K$ be strongly $\eta$-pseudomonotone. If Conditions 2.6 and 2.21 hold, then $F$ is strongly pseudo- $\eta$-invex function.

Theorem 3.8. Let the differential $F^{\prime}(u)$ of a function $F(u)$ on the invex set $K$ be strongly $\eta$-pseudomonotone. If Conditions 2.7 and 2.22 hold, then $F$ is strongly pseudo- $\eta$-invex function.

Theorem 3.9. Let the differential $F^{\prime}(u)$ of a function $F(u)$ on the invex set $K$ be $\eta$-pseudomonotone. If Conditions 2.7 and 2.22 hold, then $F$ is pseudoinvex function.

Theorem 3.10. Let the differential $F_{\varphi}^{\prime}(u)$ of a differentiable $\varphi$-preinvex function $F(u)$ be Lipschitz continuous on the $\varphi$-invex set $K$ with a constant $\beta>0$. If Condition 2.6 holds, then

$$
F(v)-F(u) \leq\left\langle F_{\varphi}^{\prime}(u), \eta(v, u)\right\rangle+\frac{\beta}{2}\|\eta(v, u)\|^{2}, \quad \forall u, v \in K
$$

Proof. for all $u, v \in K, t \in[0,1], u+t e^{i \varphi} \eta(v, u) \in K$, since $K$ is a $\varphi$-invex set. Now we consider the function

$$
\varphi(t)=F\left(u+t e^{i \varphi} \eta(v, u)\right)-F(u)-t\left\langle F_{\varphi}^{\prime}(u), \eta(v, u)\right\rangle,
$$

from which it follows that $\varphi(0)=0$ and

$$
\varphi^{\prime}(t)=\left\langle F_{\varphi}^{\prime}\left(u+t e^{i \varphi} \eta(v, u)\right), \eta(v, u)\right\rangle-\left\langle F_{\varphi}^{\prime}(u), \eta(v, u)\right\rangle .
$$

Integrating (3.29) between 0 and 1 , we have

$$
\begin{aligned}
\varphi(1) & =F\left(u+e^{i \varphi} \eta(v, u)\right)-F(u)-\left\langle F_{\varphi}^{\prime}(u), \eta(v, u)\right\rangle \\
& \leq \int_{0}^{1}\left|\varphi^{\prime}(t)\right| d t \\
& =\int_{0}^{1}\left|\left\langle F_{\varphi}^{\prime}\left(u+t e^{i \varphi} \eta(v, u)\right), \eta(v, u)\right\rangle-\left\langle F_{\varphi}^{\prime}(u), \eta(v, u)\right\rangle\right| d t \\
& \leq \beta \int_{0}^{1} t\|\eta(v, u)\|^{2} d t \\
& =\frac{\beta}{2}\|\eta(v, u)\|^{2},
\end{aligned}
$$

which implies that

$$
F\left(u+e^{i \varphi} \eta(v, u)\right)-F(u) \leq\left\langle F_{\varphi}^{\prime}(u), \eta(v, u)\right\rangle+\frac{\beta}{2}\|\eta(v, u)\|^{2},
$$


from which, using Condition 2.6, we obtain

$$
F(v)-F(u) \leq\left\langle F_{\varphi}^{\prime}(u), \eta(v, u)\right\rangle+\frac{\beta}{2}\|\eta(v, u)\|^{2}
$$

Remark 3.11. For $\eta(v, u)=v-u$ and $\alpha(v, u)=1$, the $\alpha$-invex set $K$ becomes a convex set, and consequently Theorem 3.10 reduces to the well-known result in convexity, see [12].

Using the technique of Noor [4], one can easily show that the minimum of the $\varphi$ preinvex (invex) function on the $\varphi$-invex set $K$ can be characterized by a class of variational inequalities known as $\varphi$-variational-like inequalities, that is, the minimum $u \in K$ of the $\varphi$-preinvex function $F$ is equivalent to finding $u \in K$ such that

$$
\left\langle F_{\varphi}^{\prime}(u), \eta(v, u)\right\rangle \geq 0, \quad \forall v \in K .
$$

Inequality of the type (3.33) is called the $\varphi$-variational-like inequality. In the definition of a $\varphi$-variational-like inequality problem, the underlying set is always a $\varphi$-invex set, otherwise $\varphi$-variational-like inequalities are not well defined. To the best of our knowledge, KKM theorem and diagonal convexity results cannot be used to study the $\varphi$-variationallike inequalities. It is worth mentioning that KKM theorem only holds for convex sets. It is still an open problem to prove similar results for $\varphi$-invex functions and $\varphi$-invex sets.

Theorem 3.12. Let $K$ be an invex set in $H$. Let the operator $T$ be $\eta$-pseudomonotone and $\eta$-hemicontinuous. If Condition 2.21 holds, then $u \in K$ satisfies

$$
\langle T u, \eta(v, u)\rangle \geq 0, \quad \forall v \in K,
$$

if and only if $u \in K$ satisfies

$$
\langle T v, \eta(u, v)\rangle \leq 0, \quad \forall v \in K
$$

Proof. Let $u \in K$ be such that

$$
\langle T u, \eta(v, u)\rangle \geq 0, \quad \forall v \in K,
$$

which implies that

$$
\langle T v, \eta(u, v)\rangle \leq 0, \quad \forall v \in K,
$$

since $T$ is $\eta$-pseudomonotone.

Conversely, let (3.35) hold. Since $K$ is a $\varphi$-invex set, for all $u, v \in K, t \in[0,1], v_{t}=$ $u+t e^{i \varphi} \eta(v, u) \in K$. Taking $v=v_{t}$ in (3.35) and using Condition 2.21, we have

$$
\begin{aligned}
0 & \geq\left\langle T v_{t}, \eta\left(u, u+t e^{i \varphi} \eta(v, u)\right)\right\rangle \\
& =-t\left\langle T v_{t}, \eta(v, u)\right\rangle,
\end{aligned}
$$

which implies that

$$
\left\langle T v_{t}, \eta(v, u)\right\rangle \geq 0, \quad \forall v \in K
$$


Letting $t \rightarrow 0$ in (3.39), since $T$ is $\eta$-hemicontinuous, we have

$$
\langle T u, \eta(v, u)\rangle \geq 0, \quad \forall v \in K,
$$

the required result (3.34).

Remark 3.13. Variational-like inequality (3.35) is known as Minty's variational-like inequality. $\varphi$-variational-like inequality (3.35) is also called the dual variational-like inequality. For $\varphi=0$ and $\eta(v, u)=v-u$, the invex set $K$ becomes a convex set and Theorem 3.12 reduces to Minty's lemma in variational inequalities theory. Thus, Theorem 3.12 can be viewed as a generalization of the well-known Minty's lemma.

\section{Conclusion}

In this paper, we have defined and introduced some new concepts of $\varphi$-preinvex ( $\varphi$-invex) functions and $\varphi \eta$-monotone operators. We have studied some new relationships among various concepts of $\varphi$-preinvex ( $\varphi$-invex) functions. We have tried to point out some errors that appeared in $[4,6-11]$. We have also suggested some modifications. As special cases, one can obtain correct and refined versions of the previously known results. It is an open problem to extend the KKM theorem for the $\varphi$-preinvex and $\varphi$-invex sets. This is another direction for future research work in this fascinating and dynamic field.

\section{Acknowledgment}

This research is supported by the Higher Education Commission, Pakistan, through Research Grant no. i-28/HEC/HRD/2005/90.

\section{References}

[1] M. A. Hanson, On sufficiency of the Kuhn-Tucker conditions, Journal of Mathematical Analysis and Applications 80 (1981), no. 2, 545-550.

[2] S. R. Mohan and S. K. Neogy, On invex sets and preinvex functions, Journal of Mathematical Analysis and Applications 189 (1995), no. 3, 901-908.

[3] M. A. Noor, Nonconvex functions and variational inequalities, Journal of Optimization Theory and Applications 87 (1995), no. 3, 615-630.

[4] __ On generalized preinvex functions and monotonicities, JIPAM. Journal of Inequalities in Pure and Applied Mathematics 5 (2004), no. 4, 1-9, article 110.

[5] __ Some new classes of nonconvex functions, Nonlinear Functional Analysis and Its Applications 11 (2006).

[6] G. Ruiz-Garzón, R. Osuna-Gómez, and A. Rufián-Lizana, Generalized invex monotonicity, European Journal of Operational Research 144 (2003), no. 3, 501-512.

[7] S. Schaible, Generalized monotonicity — concepts and uses, Variational Inequalities and Network Equilibrium Problems (Erice, 1994) (F. Giannessi and A. Maugeri, eds.), Plenum, New York, 1995, pp. 289-299.

[8] T. Weir and B. Mond, Pre-invex functions in multiple objective optimization, Journal of Mathematical Analysis and Applications 136 (1988), no. 1, 29-38.

[9] X. Q. Yang, Generalized convex functions and vector variational inequalities, Journal of Optimization Theory and Applications 79 (1993), no. 3, 563-580. 
[10] X. M. Yang, X. Q. Yang, and K. L. Teo, Generalized invexity and generalized invariant monotonicity, Journal of Optimization Theory and Applications 117 (2003), no. 3, 607-625.

[11] Criteria for generalized invex monotonicities, European Journal of Operational Research 164 (2005), no. 1, 115-119.

[12] D. L. Zhu and P. Marcotte, Co-coercivity and its role in the convergence of iterative schemes for solving variational inequalities, SIAM Journal on Optimization 6 (1996), no. 3, 714-726.

Muhammad Aslam Noor: Department of Mathematics, COMSATS Institute of

Information Technology, Plot 30, Sector H-8, Islamabad, Pakistan

E-mail address: noormaslam@hotmail.com

Khalida Inayat Noor: Department of Mathematics, COMSATS Institute of Information Technology, Plot 30, Sector H-8, Islamabad, Pakistan

E-mail address: khalidanoor@hotmail.com 


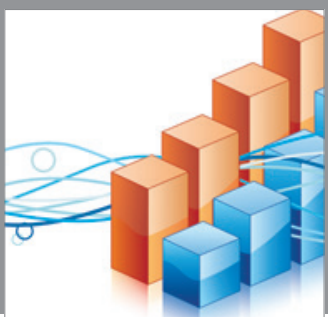

Advances in

Operations Research

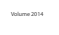

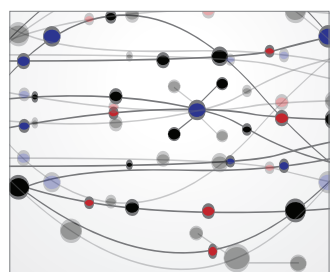

\section{The Scientific} World Journal
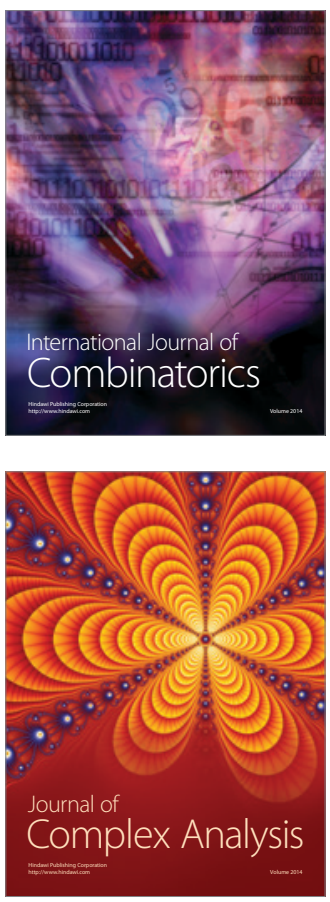

International Journal of

Mathematics and

Mathematical

Sciences
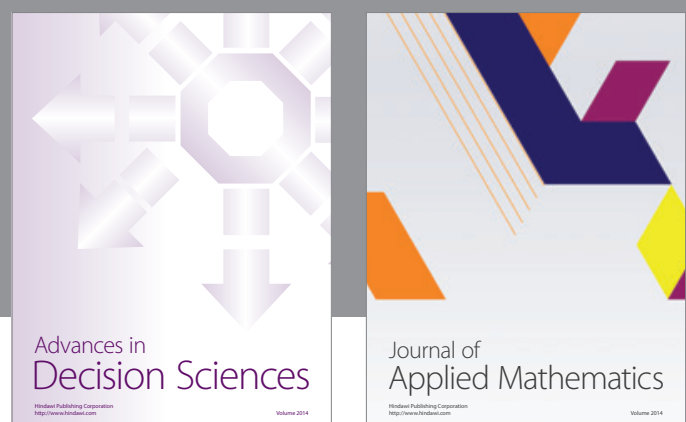

Journal of

Applied Mathematics
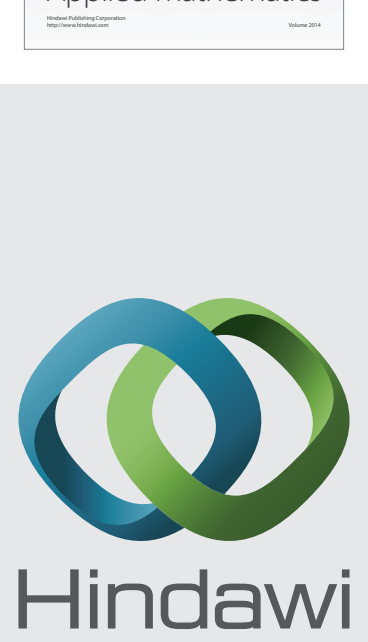

Submit your manuscripts at http://www.hindawi.com
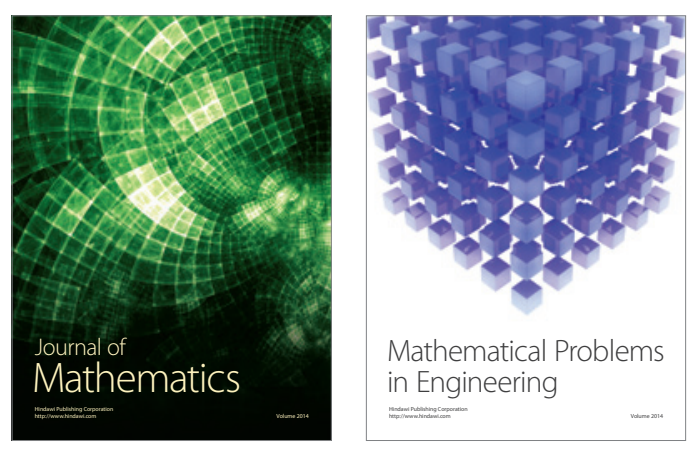

Mathematical Problems in Engineering
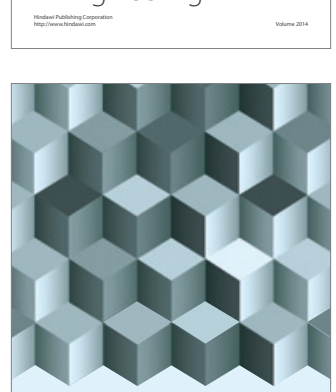

Journal of

Function Spaces
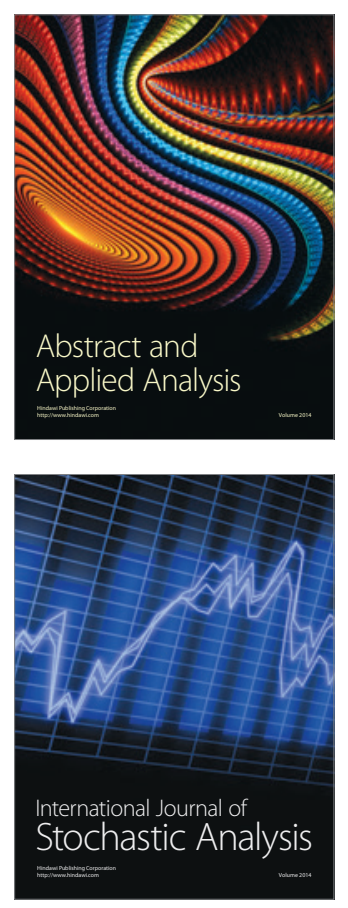

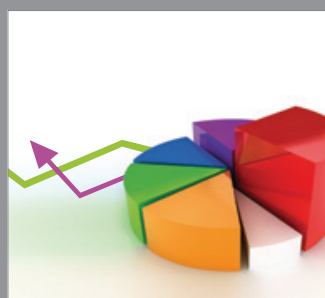

ournal of

Probability and Statistics

Promensencen
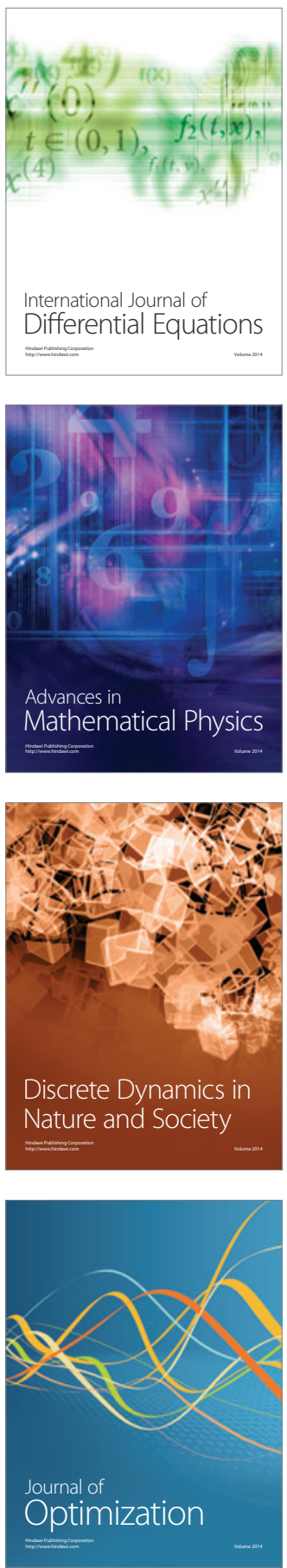